The Astrophysical Journal, 272:551-562, 1983 September 15

(1) 1983. The American Astronomical Society. All rights reserved. Printed in U.S.A.

\title{
INTERSTELLAR POLARIZATION, GRAIN GROWTH, AND ALIGNMENT
}

\author{
Per A. Aannestad \\ Department of Physics, Arizona State University \\ AND \\ J. MAYo GreEnberG \\ Huygens Laboratorium, Rijksuniversiteit Leiden \\ Received 1982 November 17; accepted 1983 March 8
}

\begin{abstract}
We show that the observed correlation of the width of the normalized linear polarization curve with the wavelength of maximum polarization is most likely caused by the accretion of mantles on grains with an initial power-law size distribution. Calculations assuming Purcell's suprathermal alignment mechanism with the "rocket" lifetime determined by a monolayer accretion time scale lead to (1) an initial steep decrease in width with increasing $\lambda_{\max }$, (2) an increase in width with intermediate values of $\lambda_{\max }$, and (3) a decrease in width for the largest values of $\lambda_{\max }$. The predicted correlation is consistent with the observed values. The Davis-Greenstein thermal alignment mechanism cannot account for the observed correlation at large values of $\lambda_{\max }$.

We have also investigated the effect of subsonic turbulent coagulation on a power-law size distribution of grains. The predicted correlation between width and $\lambda_{\max }$ is at variance with the observed data.
\end{abstract}

Subject headings: interstellar: matter - polarization

\section{INTRODUCTION}

Observations of interstellar linear polarization show a broad maximum in the region between 0.3 and $1 \mu \mathrm{m}$. For observations limited to the diffuse cloud medium, the wavelength of maximum polarization $\lambda_{\max }$ is usually well confined between 0.5 and $0.6 \mu \mathrm{m}$, and the normalized polarization curve follows the empirical curve (Serkowski 1973; Coyne, Gehrels, and Serkowski 1974)

$$
\frac{P(\lambda)}{P_{\max }}=\exp \left[-K \ln ^{2}\left(\frac{\lambda}{\lambda_{\max }}\right)\right]
$$

with the constant $K \approx 1.15$. However, for measurements covering a larger variety of regions, it is found that $\lambda_{\max }$ is generally larger in the denser regions. Furthermore, the value of $K$ appears to be an increasing function of $\lambda_{\max }$, which implies a decrease in the width of the polarization curve with increasing $\lambda_{\max }$. This effect was first noted by Codina-Landaberry and Magalhaes (1976), and Wilking et al. (1980) found from their own observations fitted to equation (1) that $K \approx 1.7 \lambda_{\max }(\mu \mathrm{m})$. Also, in a detailed study of the dark cloud R CrA, Vrba, Coyne, and Tapia (1981) find that, in the outer regions of this cloud, $\lambda_{\max }$ is well correlated with extinction and with the gas density. In this region, the proportionality of $\lambda_{\text {max }}$ to density as inferred from total extinction joins with the values typical for the diffuse medium. It should be emphasized that equation (1) with either constant or $\lambda_{\max }$-dependent $K$ is an empirical relation for which only a qualitative theoretical justification may be given (Greenberg 1978). The form of this equation just happens to give a fair fit to the actual relationships that we shall explore here.

The observational material therefore strongly suggests that the properties of the polarizing particles, be they size, shape, composition, and/or alignment efficiency, vary systematically from region to region. The observed correlation of the width of the normalized polarization curve with $\lambda_{\max }$ offers a challenge to any theory of how grains differ from one region to another. In this work we shall calculate polarization curves for size distributions of dust particles which are assumed to evolve by accretion of gaseous material onto the grain surfaces under various assumptions about the alignment mechanism and efficiency. In an earlier paper, Aannestad (1982) has made a limited number of calculations in the birefringent sphere approximation (Greenberg 1968, 1978). We shall here represent the particles as infinite cylinders, and, in addition, we shall take particle spin and disalignment into account. Properly normalized infinite cylinder cross sections are much more easily calculated than those for finite (spheroidal) particles (Rogers and Martin 1979; Onaka 1980) and provide basically the same results. Finally, we shall compare the effects of accretion and coagulation on the polarization curve. 


\section{THE GRAIN PROPERTIES}

Observations of interstellar extinction and polarization imply the following general characteristics of the dust particles (for recent reviews, see Greenberg 1978; Savage and Mathis 1979): a bimodal size distribution, with $\sim 0.1 \mu \mathrm{m}$ radii particles providing the visible extinction and polarization and much smaller particles of radii $\leqslant 0.01 \mu \mathrm{m}$ which are needed to account for the far-UV extinction. In addition, there may also be a third component of particles (e.g., graphite) to account for the presence of the $0.22 \mu \mathrm{m}$ "hump" in the extinction curve. The observation that the interstellar circular polarization curve changes sign close to $\lambda_{\max }$ is easily accounted for if the polarizing particles are made of dielectric material (Martin 1973). However, specific absorbing materials like magnetite will also satisfy this constraint (Shapiro 1975). Other constraints, such as cosmic abundances and the observed degree of polarization to extinction, are not so readily satisfied. Dielectric grains are consistent with the observed presence of silicates and ices as inferred from infrared absorption features at 10 and $3 \mu \mathrm{m}$. We shall assume that the polarizing grains are dielectric in nature and that the core particles are representative of silicates with the accreting material being similar in dielectric character to icy material.

The initial size distribution of the core particles is taken here to follow a power law:

$$
f(a)=f_{0} a^{-q}, \text { for } a_{\min } \leq a \leq a_{\max } .
$$

Such a distribution, with $q \approx 3.5$, may be formed by the collision and fragmentation processes in the atmospheres of red giants (Biermann and Harwit 1980). Although it is also the grain size spectrum that fits the average interstellar extinction and polarization curves of the silicate-graphite mixture proposed by Mathis, Rumpl, and Nordsieck (1977) (with $q=3.5$ for spheres, $q=2.5$ for infinite cylinders; Mathis 1979), it is not being used here for that purpose. In fact, the larger graphite particles in the silicate-graphite mixture appear to be excludable on observational grounds (Greenberg and Chlewicki 1983). In our case we shall not include any graphite particles. We shall allow the power-law index $q$ for infinite cylinders to vary in the range $2-3$, so as to best fit the observed large polarization widths for the smallest values of $\lambda_{\max }$. The size range $a_{\min }$ to $a_{\max }$ is similarly chosen to reproduce the smallest observed values of $\lambda_{\max }=0.35 \mu \mathrm{m}$, thus assuming that for these strong deviations from the normal diffuse cloud properties the grain mantles may be negligibly small.

Interstellar grains may grow in size by simple accretion of gaseous atoms and molecules in a mantle or perhaps by coagulation during grain-grain collisions. The conditions under which these processes may occur, and the corresponding growth rates, are quite uncertain, but this shall not concern us here. We shall assume that an initial size distribution of grains (ejected from stellar sources) experience growth sometime during their residence in interstellar clouds (Greenberg 1982). Our work is concentrated on the effects of mantle accretion on the polarization curve, but we have also made some calculations for a simple case of growth by turbulent coagulation in a dense $\left(\sim 10^{4} \mathrm{~cm}^{-3}\right)$ cloud.

If the grains accrete mantles, the size distribution at any time $t$ is derived from the initial size distribution according to

$$
\begin{aligned}
f(a, t)= & f_{0}(a-\alpha t)^{-q}=f_{0}(a-\Delta a)^{-q}, \\
& \text { for } a_{\min , 0}+\alpha t \leq a \leq a_{\max , 0}+\alpha t,
\end{aligned}
$$

where $\alpha=d a / d t=$ constant, so that the mantle thickness $\Delta a$ is the same on all grains. It is assumed that the very small grains implied by the UV extinction observations do not similarly grow mantles. This may be due to temperature fluctuations from absorption of UV photons (Greenberg and Hong 1974; Purcell 1976), surface desorption by localized phonons (Greenberg 1979), or from molecule formation on the surface (Allen and Robinson 1975). Fluctuations due to the unshielded interstellar radiation field may naturally tend to form a bimodal size distribution of interstellar grains (Aannestad and Kenyon 1979). Here we investigate the implications of such a model for interstellar polarization observations.

\section{POLARIZATION AND ALIGNMENT}

For a size distribution of cylinders of constant elongation $e$ (ratio of length to diameter) that have accreted mantles of thickness $\Delta a$ we write the cross section for linear polarization as given by

$$
C_{\mathrm{pol}}=4 e \int_{a_{\min }}^{a_{\max }} a^{2} f(a, \Delta a) Q_{\mathrm{pol}}[\bar{\beta}(a)] d a,
$$

where $\bar{\beta}(a)$ is a mean precession cone angle between the magnetic field and the grain minor axis for a grain of total size $a$. By definition, $\beta=0$ for perfect spinning alignment (PSA). The function $Q_{\mathrm{pol}}(\bar{\beta})$ is calculated by an integration over spin angle and precession angle as given by Hong and Greenberg (1980, eq. [4]). We shall define $\bar{\beta}$ via $\cos ^{2} \bar{\beta}=\left\langle\cos ^{2} \beta\right\rangle$, and its value depends on the alignment mechanism. If we define thermal spinning alignment (TSA) as that which is governed by a kinetic balance between the magnetic dissipation torques (Davis and Greenstein 1951) and the gas collisional disalignment (see Jones and Spitzer 1967; Greenberg 1968), one can show that

$$
\overline{\cos ^{2} \beta}=\left(\frac{1}{1-\xi^{2}}\right)\left[1-\frac{\xi}{\left(1-\xi^{2}\right)^{1 / 2}} \sin ^{-1} \frac{1-\xi^{2}}{\left(1-\xi^{2}\right)^{1 / 2}}\right],
$$


where the alignment parameter $\xi$ is defined by

$$
\xi^{2}=\frac{1+\delta_{\mathrm{TA}}\left(T_{d} / T\right)}{1+\delta_{\mathrm{TA}}},
$$

and

$$
\delta_{\mathrm{TA}}=\left(\frac{\chi^{\prime \prime}}{\omega}\right)\left(\frac{B^{2}}{2 a n_{\mathrm{H}}}\right)\left(\frac{2 \pi}{m_{\mathrm{H}} k T}\right)^{1 / 2}
$$

Here $T_{d}$ is the dust temperature, $T$ is the gas temperature, $\chi^{\prime \prime} / \omega$ is the ratio of the imaginary part of the magnetic susceptibility to the angular rotation frequency of the grain, $B$ is the magnetic field strength, and the gas is composed of particles of mass $m_{\mathrm{H}}$ with a density $n_{\mathrm{H}}$. In equation (7) a roughly spherical grain has been assumed. If we set $\chi^{\prime \prime} / \omega=2.5 \times 10^{-12} / T_{d}$ (Jones and Spitzer 1967) and let $m_{\mathrm{H}}$ be the mass of a hydrogen atom,

$$
\begin{aligned}
\delta_{\mathrm{TA}}= & 0.21\left(\frac{10 \mathrm{~K}}{T_{d}}\right)\left(\frac{B}{1 \mu \mathrm{G}}\right)^{2}\left(\frac{1 \mathrm{~cm}^{-3}}{n_{\mathrm{H}}}\right) \\
& \times\left(\frac{100 \mathrm{~K}}{T}\right)\left(\frac{0.1 \mu \mathrm{m}}{a}\right) .
\end{aligned}
$$

With reasonable values for the interstellar parameters as indicated in equation (8), $\delta_{\mathrm{TA}}=0.21$, corresponding to $\xi^{2}=0.84$ or a mean cone angle of $\sim 50^{\circ}$. This implies only a slight degree of alignment. For elongated grains the value of $\delta_{\mathrm{TA}}$ is even smaller (roughly, $\delta_{\mathrm{TA}} \propto 1 / e$ ), leading to the same large cone angle. From scattering experiments and calculations (Greenberg 1968; Rogers and Martin 1979) it appears that a value of $e>2$ is required to provide the observed amount of polarization. Jones and Spitzer (1967) discussed the possibility that the grain is superparamagnetic with an enhanced value of $\chi^{\prime \prime}$, and in the following we shall assume that an enhancement by a factor of $10-100$ is possible (or $10 e-100 e$ for an elongation of $e$ ). Alternatively, we may assume a higher magnetic field by the corresponding factor. We shall further assume, for simplicity, that $B \propto n_{\mathrm{H}}^{0.5}$. This is in the range of the relations expected for contracting interstellar clouds (Mouschovias 1978). Neglecting any other variations in the parameters from their typical values (which would be small anyway relative to the uncertainty in $\left.\chi^{\prime \prime}\right)$, the mean cone angle $\bar{\beta}(a)$ is determined by equations (5) and (6).

An alternative and stronger alignment mechanism has been proposed by Purcell (1979), who shows how irregularities in the shape of surface characteristics of a grain may cause suprathermal rotation by the "rocket effect" which then aligns the grain more effectively via the magnetic dissipation (paramagnetic relaxation) torques than is possible with the usual thermal treatment of the
Davis-Greenstein mechanism. In this "suprathermal" spinning alignment (SSA) mechanism the mean degree of alignment $\bar{Q}_{A}=\left(\frac{3}{2}\left\langle\cos ^{2} \beta\right\rangle-\frac{1}{2}\right)$ is given by

$$
\bar{Q}_{A}=\bar{\delta}_{\mathrm{SA}}^{-1} \int_{0}^{\infty} Q_{A}(\delta) \exp \left(-\delta / \bar{\delta}_{\mathrm{SA}}\right) d \delta,
$$

where

$$
Q_{A}(\delta)=\frac{3}{2} \frac{1-\exp (\delta-1) \tan ^{-1}[\exp (\delta)-1]}{1-\exp (-\delta)}-\frac{1}{2},
$$

and $\bar{\delta}_{\mathrm{SA}}=2 t_{x} / t_{r}$, with $t_{x}$ the mean time between disalignments $\left(Q_{A}=0\right)$ and $t_{r}$ the paramagnetic alignment time. We note here that the quantity $Q_{A}$ is equal to the Rayleigh reduction factor, which is the reduction in the degree of polarization relative to PSA for small particles satisfying the Rayleigh approximation (Greenberg 1968, 1969). According to Purcell (1979) the ratio $t_{x} / t_{r}$ is approximately given by $t_{x} / t_{r} \approx 1.3\left(1+t_{L} / t_{d}\right)\left(t_{d} / t_{r}\right)$, where $t_{L}$ is the correlation time for the effective torques and $t_{d}$ is the rotational damping time.

Since we are considering grains that grow by mantle accretion, the time $t_{L}$ cannot be larger than the time it takes for a "new" layer to form. If $\Delta h$ is the thickness of this layer, we shall set $t_{L}=\Delta h /(d a / d t)$ so that

$$
\frac{t_{L}}{t_{d}}=\frac{3}{\left(t_{d} / t_{m}\right)} \Delta h\left(\frac{m_{\mathrm{H}}}{m_{a}}\right)^{1 / 2}\left(\frac{n_{\mathrm{H}}}{n_{a}}\right) \frac{1}{a},
$$

where the accreting particles have a mass $m_{a}$ and a density $n_{a}$, and $t_{m}$ is the time required for the grain to be struck by a mass of gas particles $\left(m_{\mathrm{H}}\right)$ equal to its own mass. In equation (11) we have set the ratio of the mean density of the accreting grain equal to the mean density in the accreted layer. For cylinders of constant elongation this ratio varies between 0.4 and 1.5 if the density ratio of the core material to the mantle material is 2.5. For cylinders spinning about an axis, $t_{d} / t_{m} \approx 0.5$ (Purcell and Spintzer 1971). The ratio $t_{d} / t_{r}$ is calculated by Purcell and Spitzer (1971) for various shapes of grains, and we find that $t_{d} / t_{r}=0.31 c \delta_{\mathrm{TA}}$, where the factor $c$ varies from 1.8 to 0.7 for spheroids of elongation 2 to 4 . We shall use $c=1.3$ for all of our particles. The basic ratio $t_{x} / t_{r}$ is therefore

$$
\frac{t_{x}}{t_{r}} \approx 0.52\left[1+6 \Delta h\left(\frac{m_{\mathrm{H}}}{m_{a}}\right)^{1 / 2}\left(\frac{n_{\mathrm{H}}}{n_{a}}\right) \frac{1}{a}\right] \delta_{\mathrm{TA}} .
$$

For the thickness $\Delta h$ of the layer that effectively covers the rocket sites we shall assume $3.7 \AA$, which is an average molecular diameter for simple molecules. The number of accreting gas particles is limited by the total 
amount of heavy atoms in the gas phase and will be decreasing as the grains grow their mantles. Also, some of the interstellar material may be "locked up" in other grain components than the polarizing component studied here (small particles, graphite, etc.). We shall let $n_{a} / n_{\mathrm{H}}$ $=10^{-3} d$, where $d$ is the "depletion" of the accreting material, and is initially assumed to be unity. Neglecting shape factors, we find that $d$ is given by

$$
d(\Delta a)=1-4 \times 10^{3}\left(\frac{\rho_{m}}{m_{a}}\right)\left(\frac{n_{\mathrm{gr}}}{n_{\mathrm{H}}}\right) \int_{0}^{\Delta a} \pi a_{\mathrm{eff}}^{2} d(\Delta a),
$$

where $a_{\text {eff }}$ is a mean grain radius weighted by $a^{2}, \rho_{m}$ is the mantle specific density, and $n_{\mathrm{gr}}$ is the total number density of accreting grains. We shall use the reasonable values of $\rho_{m}=1 \mathrm{~g} \mathrm{~cm}^{-3}, n_{\mathrm{gr}} / n_{\mathrm{H}}=5 \times 10^{-13}$, and $m_{a}=$ $16 m_{\mathrm{H}}$. With the value of $\delta_{\mathrm{TA}}$ as given by equation (8) for the parameters indicated, we finally have

$$
\frac{t_{x}}{t_{r}} \approx \frac{0.1}{F}\left\{1+\left[\frac{5.6}{d(\Delta a)}\right]\left[\frac{0.1 \mu \mathrm{m}}{a(\mu \mathrm{m})}\right]\right\} \frac{0.1 \mu \mathrm{m}}{a(\mu \mathrm{m})},
$$

where $F$ is introduced as a correlation factor of $\sim 0.8$ for incomplete disorientation (Spitzer and McGlynn 1979) at the time the rockets are "destroyed." Equations (9)-(14) determine the mean cone angle $\bar{\beta}$ for a grain of radius $a$ when the gas depletion is $d(\Delta a)$.

Figure 1 shows how the cone angle depends on grain size for the TSA and SSA mechanisms for various values of enhancement in the paramagnetic susceptibility. The SSA mechanism is clearly superior for sizes $\leqslant 0.1 \mu \mathrm{m}$ since even under conditions of perfect TSA $\left(\chi^{\prime \prime} \rightarrow \infty\right)$, the cone angle is limited to $\bar{\beta} \geq 36^{\circ}$, unless $T_{d} / T<0.1$. However, a much smaller value than 0.1 for the temperature ratio is unrealistic for most interstellar clouds (Greenberg 1971).

\section{GROWTH BY COAGULATION}

It is the aim of this section to obtain an approximate understanding of how coagulation may modify the finite size distribution given in equation (2) and contrast its consequences on polarization with the changes caused by size-independent accretion of grain mantles. We shall not inquire into the details of the coagulation process itself, but rather assume that whenever two grains of radii $a_{1}$ and $a_{2}$ collide, they form a new grain of volume $4 / 3 \pi\left(a_{1}^{3}+a_{2}^{3}\right)$. We shall also let such coagulation of spherical particles be representative for the coagulation of our infinite cylinders, given the clear difficulty of incorporating shape effects into the treatment, as well as the problem associated with growth inhibition by the rapid spinning of the particles.

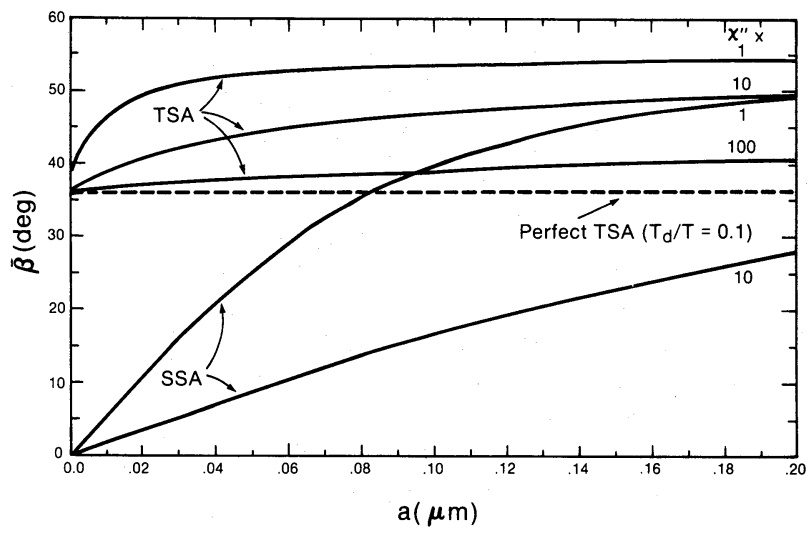

FIG. 1.- Mean precession cone angle between the direction of the magnetic field and the grain minor axis as a function of the grain radius. TSA : thermal spinning alignment (Davis-Greenstein) with enhancements in the paramagnetic susceptibility $\chi^{\prime \prime}$ by factors of 1,10 , and 100 . The dust temperature $T_{d}=10 \mathrm{~K}$, and the gas temperature $T=100 \mathrm{~K}$. Dashed curve: the case for perfect thermal alignment $\left(\delta_{\mathrm{TA}} \rightarrow \infty\right)$. SSA: suprathermal spinning alignment with enhancement factors in $\chi^{\prime \prime}$ of 1 and 10 .

However, we shall use grain-grain velocities as derived for a subsonic turbulent velocity field by Völk et al. (1980). From their expressions we find that the basic parameter for the interaction of a grain of radius $a$ with a turbulent gas with a Kolmogoroff velocity spectrum is given by the ratio

$$
\frac{t_{m}}{t_{\kappa}}=4.27 \times 10^{-2}\left(\frac{20 \mathrm{~K}}{T}\right)^{1 / 2}\left(\frac{10^{4} \mathrm{~cm}^{-3}}{n_{\mathrm{H}}}\right)\left(\frac{v}{\kappa}\right) s a(\mu \mathrm{m}),
$$

where $t_{m}$ is the time for the grain to be struck by a mass of gas equal to its own mass, $t_{\kappa}$ is the time for a turbulent cell to move across the maximum turbulent scale, $\kappa$, in units of the Jeans length when moving at an rms turbulent velocity $v$ in units of the gas thermal velocity, $s$ is the grain specific density, and $T$ and $n_{\mathrm{H}}$ are the gas temperature and density, respectively (in the work by Völk et al., $t_{m}$ and $t_{\kappa}$ are called $\tau_{f}$ and $\tau_{\kappa_{0}}$, respectively). Since interstellar clouds show turbulent velocities of the order of the thermal velocity or greater, we shall set $v$ close to unity, the value at which any theory for subsonic turbulence must break down. However, a further extension must await a theory for the effect of supersonic turbulence on grain-grain collisions. With $v=0.99, \kappa=1, s=2.5 \mathrm{~g} \mathrm{~cm}^{-3}, n_{\mathrm{H}}=10^{4} \mathrm{~cm}^{-3}$, and $T=20 \mathrm{~K}$, Figure 2 shows the turbulent relative velocity $V_{\text {rel }}\left(a_{1}, a_{2}\right)$ between two grains of radii $a_{1}$ and $a_{2}$ in the range 0.01 to $0.35 \mu \mathrm{m}$. The velocities are of the order of $0.1 \mathrm{~km} \mathrm{~s}^{-1}$, and agree with the approximate values derived by Scalo (1977) for nonidentical particles subject to inertial turbulent coagulation. We shall assume that neither vaporization nor shattering take place 


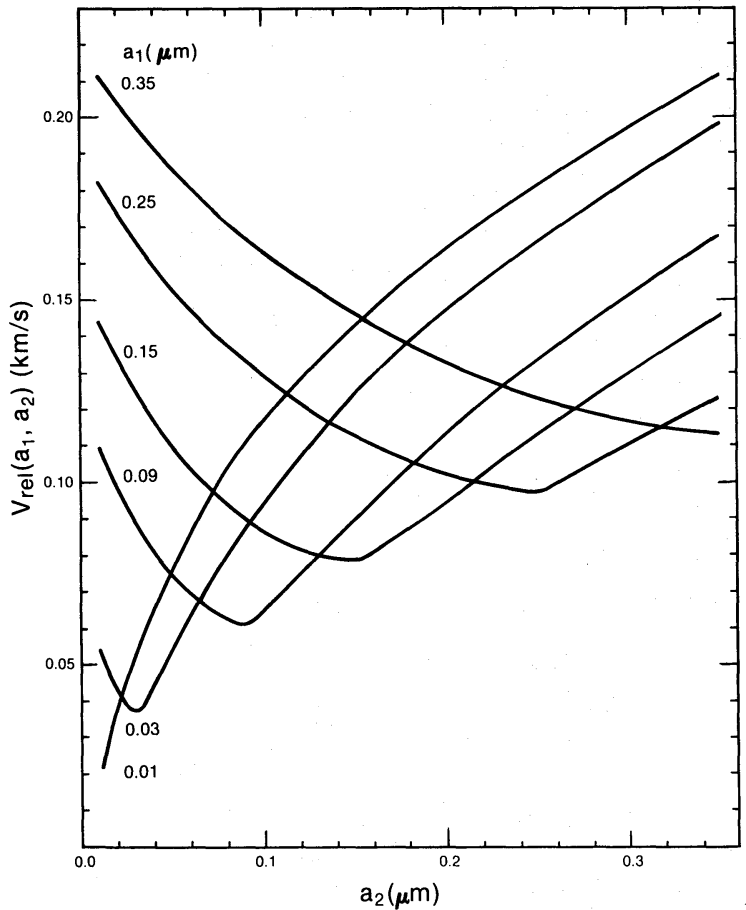

FIG. 2. - The relative velocity between two grains of radii $a_{1}$ and $a_{2}$ as calculated from the expressions given by Völk et al. (1980) for a subsonic turbulent velocity field. The rms gas turbulent velocity is 0.99 times the thermal velocity, the gas temperature and density are $20 \mathrm{~K}$ and $10^{4} \mathrm{~cm}^{-3}$, respectively, the grain specific density is $2.5 \mathrm{~g} \mathrm{~cm}^{-3}$, and the maximum turbulent scale equals the Jean's length.

at these velocities; this depends on the physical strength of the material and perhaps also on the grain shapes and their spin (particularly for suprathermal spin-up). For spherical grains of radius $0.1 \mu \mathrm{m}$ with a lattice bond strength of $3 \mathrm{eV}$, complete vaporization occurs for relative velocities above $\sim 10 \mathrm{~km} \mathrm{~s}^{-1}$, and the limit for shattering is $\sim 0.5 \mathrm{~km} \mathrm{~s}^{-1}$ (Scalo 1977). Any grain charge that could decrease the "sticking" efficiency of two grains is neglected, but, on the other hand, we shall also neglect any increase due to induced Van der Waals forces. With these assumptions the coagulation equation in normalized and discretized form is

$$
\begin{aligned}
\frac{\Delta Y_{i}}{\Delta \tau}= & -Y_{i} \sum_{j=1}^{j_{\max }} C_{i j} Y_{j}+\frac{1}{2} \sum_{j \neq i-j}^{i-1} C_{i-j, j} Y_{i-j} Y_{j} \\
& +C_{i / 2, i / 2} Y_{i / 2}^{2},
\end{aligned}
$$

where the last term is counted only when $i$ is even, and $C_{i j}$ in the loss term is multiplied by a factor of 2 when $i=j$. In this equation $Y_{i}=f_{i}(t) \Delta V_{i} / N_{0}$, where $f_{i} \Delta V_{i}$ is the number of grains of radius $a_{i}$ in the volume interval $\Delta V_{i}$ and $N_{0}=\sum_{i} f_{i} \Delta V_{i}$ at time $t=0, \tau=t / t_{c}$, where $t_{c}=\left(N_{0} \pi a_{0}^{2} V_{\mathrm{rms}}\right)^{-1}, a_{0}$ is the radius of a particle of unit volume, and $V_{\mathrm{rms}}$ is the gas rms turbulent velocity. The kernel in the coagulation equation is

$$
C_{i j}=\frac{\left(a_{i}+a_{j}\right)^{2}}{a_{0}^{2}} \frac{V_{\mathrm{rel}}\left(a_{i}, a_{j}\right)}{V_{\mathrm{rms}}} .
$$

In addition, volume conservation requires that $\sum_{i} V_{i} f_{i} \Delta V_{i}$ $=$ constant.

Equation (16) has been solved in a variable-step Runge-Kutta procedure for a size distribution $f\left(a_{i}\right)=$ $f_{0} a_{i}^{-3}$ and an initial range in radii of $0.04-0.2 \mu \mathrm{m}$. Computer storage requirements made us use a slightly larger minimum size than the $0.03 \mu \mathrm{m}$ size in the earlier calculations, but we shall compare with size-independent accretion of the same, slightly narrower size distribution $(\S \mathrm{V})$. The initial number of interacting sizes was $(0.2 / 0.04)^{3}=125$, and in addition 50 empty bins were added for a total of 175 size bins. Whenever volume conservation was violated by more than $5 \%$ the loss was made up by adding new bins with number densities extrapolated as a power law fitted to the size distribution, for up to a maximum of 400 interacting sizes. When this maximum number was reached, the step size was increased by a factor of 4, defining 100 new interacting size bins. However, in such a "compression" the smallest grains in the size distribution are necessarily "lost." We therefore decided to "save" before every compression the first three size bins and for these sizes $Y_{i}^{\prime}$ solve the loss equation

$$
\frac{\Delta Y_{i}^{\prime}}{\Delta \tau}=-Y_{i}^{\prime} \sum_{j=i}^{j_{\max }^{\prime}} C_{i j} Y_{j}^{\prime}
$$

where $Y_{j}^{\prime}$ are the current values (before the step $\Delta \tau$ ) of both the "saved" and the main values and $\Delta \tau$ equals the step in the evolution of the main array (eq. [16]). We have thus neglected gain terms for these smaller grains but also, as a compensating factor, neglected some loss terms by requiring $j \geq i$. Clearly, this is not a very accurate procedure, and for time intervals $\tau \geq 1$ it tends to let the smaller grains "break away" from the main distribution. We believe, however, that since we are interested in the initial modifications of the size distribution $(\tau \leq 1)$ the present treatment is adequate. Figure 3 shows the development of the power-law size distribution for the same parameters as assumed in Figure 2. We note that the distribution becomes less steep only relatively slowly, and that the number of large grains start to build up when $\tau \geqslant 0.5$. The steepness of the distribution changes from $a^{-3}$ to an average of $\sim a^{-1.8}$ as $\tau$ goes from 0 to 0.5 . For comparison we also show the steepening of the size distribution if the grains are subject to size-independent accretion of mantles. For a mantle thickness of 0.06 and $0.15 \mu \mathrm{m}$ the equivalent power-law index is increased to $\sim 5$ and 10 , respectively. 


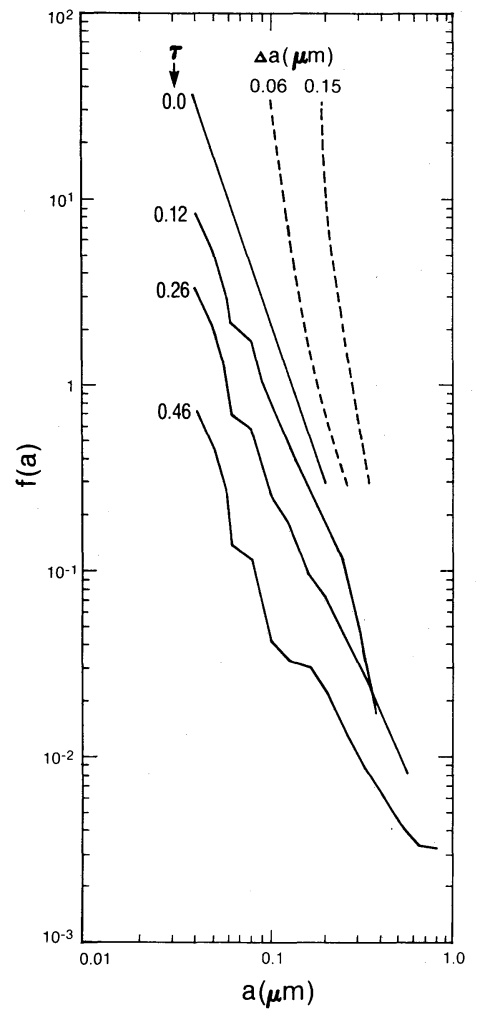

FIG. 3.- Solid curves: the numerical evolution of a finite power-law size distribution of grains subject to subsonic turbulent coagulation. The quantity $\tau$ is a nondimensional time parameter, and other parameters are as in Fig. 2. For comparison, dashed curves: the steepening of the power-law distribution when subject to mantle accretion with mantle thickness $\Delta a$.

\section{CALCULATIONS}

The linear polarization (eq. [4]) has been calculated for $\sim 30$ wavelengths in the range near-UV to nearinfrared, assuming a constant refractive index $\left(m=m^{\prime}\right.$ $\left.-i m^{\prime \prime}\right)$ for the core as well as the mantle material. We have used $m_{\text {core }}=1.6-0.018 i, m_{\text {mantle }}=1.3-m^{\prime \prime} i$, with $m^{\prime \prime}$ allowed to vary. The initial minimum and maximum grain radii are 0.03 and $0.2 \mu \mathrm{m}$, respectively. With these limits the rms grain radius equals $0.053 \mu \mathrm{m}$, which is approximately the same as the silicate core radius in the core-mantle grain model of Hong and Greenberg (1980). The number of size bins in the size distribution was 23 for the PSA case and 11 for the imperfect spinning cases, the number of spin angles was 21 and 11, respectively, and the number of precession angles for imperfect alignment was 11. Typically, on an Amdahl V7B computer, a calculation for a given size distribution would take $\sim 15$ minutes for the PSA case and $\sim 60$ minutes for the other two cases. The resulting polarization curve was normalized to its value at $\lambda_{\max }$ and the width $W$ defined as the difference in $\lambda_{\max } / \lambda$ between the half-power points of the normalized curve.
Below, we compare the results with the observational half-widths as determined by $W=\exp \left[(\ln 2 / K)^{1 / 2}\right]-$ $\exp \left[-(\ln 2 / K)^{1 / 2}\right]$, where $K\left(\lambda_{\max }\right)$ is taken from Wilking et al. (1980). (For clarity in the figures, only 10 representative points of the total of 24 observations are shown).

\section{a) Perfect Spinning Alignment}

Figure 4 shows $W\left(\lambda_{\max }\right)$ for a set of perfectly spinning cylinders with $a_{\min }=0.03 \mu \mathrm{m}+\Delta a, a_{\max }=0.2 \mu \mathrm{m}$ $+\Delta a$, a power-law index $q=3.0$, and $\Delta a$ varying from 0 to $0.15 \mu \mathrm{m}$. The initial rapid decrease in width with $\lambda_{\max }$ is caused by the rapidly decreasing width of the power-law size distribution with small increases in grain size. Once a nearly single size is reached, however, the shape of the normalized curve does not change much, and $W \approx$ constant. Figure 4 shows that the predicted width is smaller than the observational results for $\lambda_{\max }$ $\geq 0.5 \mu \mathrm{m}$, unless the imaginary part of the refractive index of the mantle material is $m^{\prime \prime} \geq 0.3$. The same conclusion holds even stronger if finite nonspherical particles are considered, since such particles have a slightly narrower polarization curve than infinite cylinders (Rogers and Martin 1979). Calculations in the

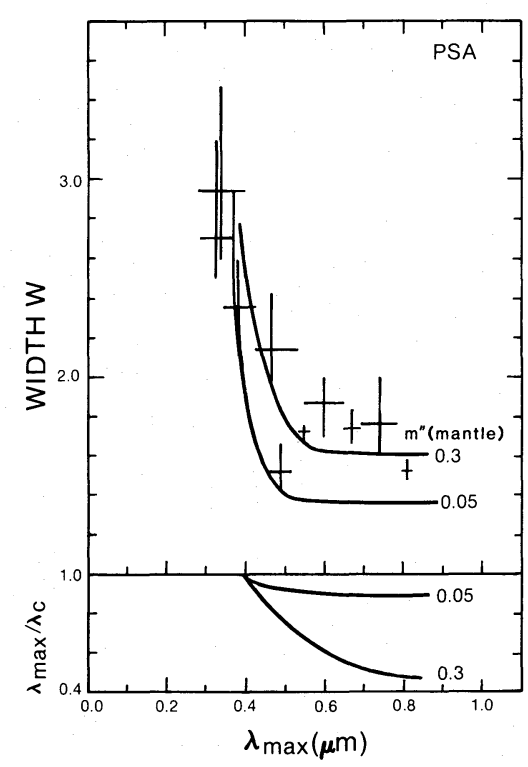

FIG. 4. - Upper part: the width of the normalized polarization curve as a function of the wavelength of maximum linear polarization when a power-law size distribution $\left(\propto a^{-3}\right)$ of perfectly spinning infinite cylinders is subject to growth by mantle accretion. The minimum and maximum initial grain sizes are 0.03 and 0.2 $\mu \mathrm{m}$, respectively. The refractive index for the core material is 1.6-0.018i, and the index for the mantle is $1.3-m^{\prime \prime} i$, with the values of $m^{\prime \prime}$ as indicated. Crosses: values deduced from the observations of Wilking et al. (1980). Lower part: the variation in the ratio of the wavelength of maximum linear polarization to the wavelength at which the circular polarization goes through zero with the wavelength of maximum polarization. 
birefringent sphere approximation lead to the requirement that $m^{\prime \prime} \geq 0.5$ (Aannestad 1982). Absorbing grains, however, will, in general, not account for the observed variation of circular polarization with wavelength. As the lower part of Figure 4 shows, the ratio of $\lambda_{\max }$ to $\lambda_{c}$, where $\lambda_{c}$ is the wavelength at which the circular polarization goes through zero, decreases to $\sim 0.5$ for the largest values of $\lambda_{\max }$ if $m^{\prime \prime}$ is as large as 0.3 . This contradicts the observational fact that $\lambda_{\max } \approx \lambda_{c}$, although we are not aware of observations of $\lambda_{c}$ in the regions of highest $\lambda_{\max }$. We shall go on to consider how, for dielectric grains, the more likely imperfect alignment mechanisms may lead to larger widths at larger $\lambda_{\max }$, in better agreement with the observations.

\section{b) Thermal Spinning Alignment}

Figure 5 shows the width of the polarization curve for wobbling infinite dielectric cylinders $\left(m^{\prime \prime}[\right.$ mantle $\left.]=0.0\right)$ subject to the thermal spinning alignment mechanism with $T_{d} / T=0.1$ and enhancement factors in the magnetic susceptibility $\chi^{\prime \prime}$ of 10 and 100 . Since less well aligned grains will show a decrease in the relative contribution to the polarization by the larger grains, $\lambda_{\max }$ will change to smaller values for a given size distribution. For the case of $\chi^{\prime \prime} \times 10$ we have compensated for this by decreasing the power-law index to $q=2.5$. The minimum and maximum initial grain sizes are 0.03 and 0.2 $\mu \mathrm{m}$, respectively.

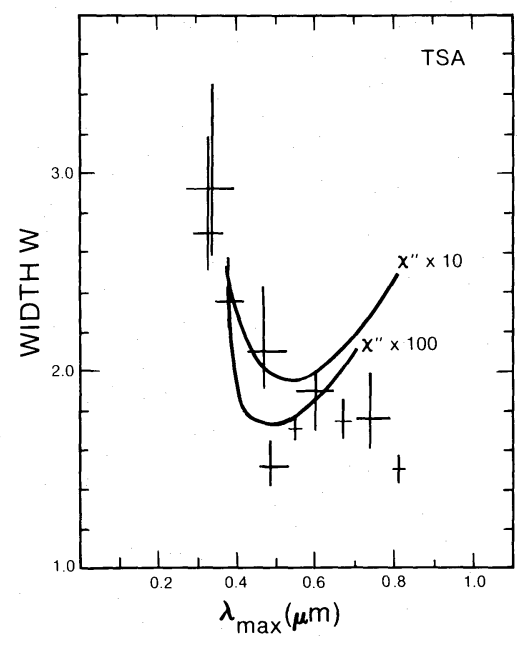

FIG. 5
The characteristic behavior of the shape of the polarization curve in the case of TSA is a pronounced minimum in the width at $\lambda_{\max }=0.5-0.6 \mu \mathrm{m}$. For smaller $\lambda_{\max }$ the decrease in the slope of the size distribution causes an increase in width, and for larger $\lambda_{\max }$ the width increases because of the increasing disalignment for larger grains. The predicted widths are too large compared to the observations at $\lambda_{\max } \gtrsim 0.6 \mu \mathrm{m}$, even for an enhancement factor in $\chi^{\prime \prime}$ of 100 .

\section{c) Suprathermal Spinning Alignment}

In Figure 6 we show the predicted variation of $W$ with $\lambda_{\max }$ for dielectric cylinders subject to Purcell's suprathermal alignment mechanism. The power-law index in the size distribution is 2.0 for the case of no magnetic enhancement $\left(\chi^{\prime \prime} \times 1\right)$ and is 2.5 for the case of enhancement by a factor of $10\left(\chi^{\prime \prime} \times 10\right)$. The unique feature about SSA is the implied decrease in width for the largest $\lambda_{\max }$ owing to the rapid depletion of accreting material. We see that with no assumed enhancement in the magnetic susceptibility this leads to a maximum in $W$ for $\lambda_{\text {max }} \approx 0.7 \mu \mathrm{m}$ and to values in much better agreement with the observations than for the case of TSA. The dashed curve shows the width if the effect of depletion is not taken into account, i.e., the disalignment time scale is a constant equal to the initial time scale. In this case, the behavior is very similar to that for TSA in Figure 5. We also show the curve for an assumed

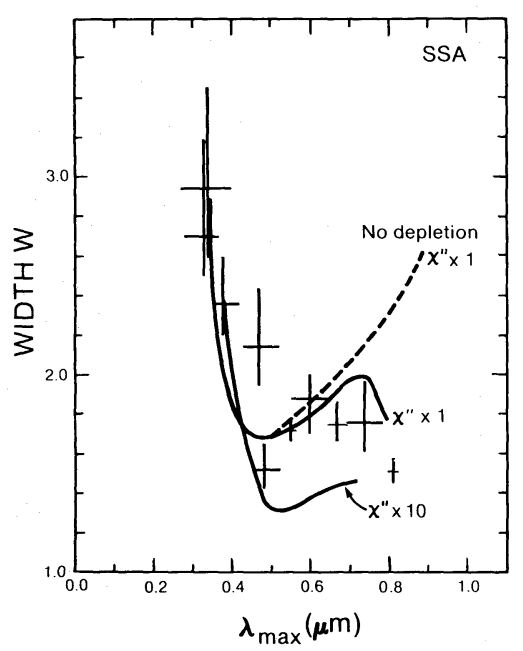

FIG. 6

FIG. 5. - The variation in the width of the normalized polarization curve with the wavelength of maximum polarization for a size distribution of spinning infinite cylinders subject to thermal alignment (Davis-Greenstein) and growth by mantle accretion. Enhancements in the imaginary part of the magnetic susceptibility $\chi^{\prime \prime}$ by factors of 10 and 100 are assumed. The refractive index for the core material is $1.6-0.018 i$, and the index for the mantle is 1.3-0.0 $i$. The power-law index in the size distribution is 2.5 for the $\chi^{\prime \prime} \times 10$ case, and 3.0 for the $\chi^{\prime \prime} \times 100$ case. The initial grain sizes are as in Fig. 4 .

FIG. 6. - The variation in the width of the normalized polarization curve with the wavelength of maximum polarization for a size distribution of spinning infinite cylinders subject to suprathermal alignment and growth by mantle accretion. The enhancement factors in the magnetic susceptibility $\chi^{\prime \prime}$ are as indicated, and the optical properties and initial grain sizes are as in Fig. 5. The power-law index in the size distribution is 2.0 for the $\chi^{\prime \prime} \times 1$ case, and 2.5 for the $\chi^{\prime \prime} \times 10$ case. Dashed curve: the variation if no depletion of the accreting material is assumed. 


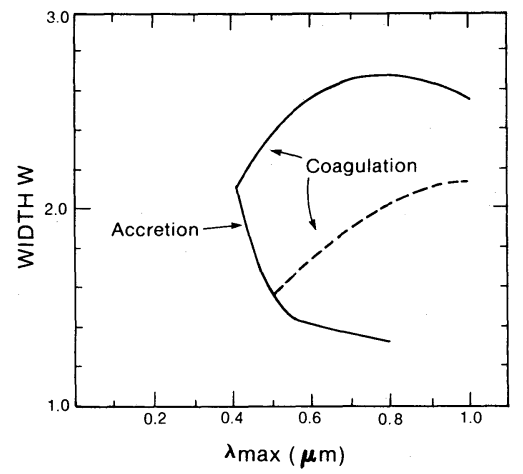

FIG. 7. - The width of the normalized polarization curve as a function of the wavelength of maximum polarization when the size distribution is subject to growth by subsonic coagulation or by mantle accretion. The grains are assumed to be perfectly spinning dielectric cylinders with a size distribution that evolves as shown in Fig. 3. Dashed curve: the variation in $W$ owing to coagulation if the size distribution is initially characterized by a power-law index of 3.5 , a minimum grain size of $0.07 \mu \mathrm{m}$, and a maximum grain size of $0.21 \mu \mathrm{m}$

enhancement in $\chi^{\prime \prime}$ of a factor of 10 . It is evident that SSA is capable of producing the observed widths at large $\lambda_{\max }$ with no, or only a moderate (factor $<10$ ), increase in $\chi^{\prime \prime}$.

\section{d) Effect of Coagulation}

We assume that the coagulating particles have achieved perfect spinning alignment at all times. Figure 7 shows $W\left(\lambda_{\max }\right)$ for the particle size distribution evolving as in Figure 3 from $\tau=0$ to $\tau=0.37$. The width is characteristically at least as large as the initial width as the grains grow larger and the slope of the distribution decreases. Coagulation of small grains therefore seems unable to reproduce the observed $W\left(\lambda_{\max }\right)$. Any disalignment of the grains with increasing size would tend to make the curve even wider, thus increasing the discrepancy. For comparison, also shown in Figure 7 is the curve for size-independent accretion, assuming the same initial conditions and PSA.

Although coagulation does not fit the observations of $W$ at small $\lambda_{\max }$, it may be important in regions where $\lambda_{\max }$ is already $\gtrsim 0.5 \mu \mathrm{m}$. We have therefore assumed that a size distribution of silicate grains with radii $0.07-0.21 \mu \mathrm{m}, q=3.5$ is subject to coagulation, and the resulting $W\left(\lambda_{\max }\right)$ is shown as the dashed curve in Figure 7.

\section{DISCUSSION}

A basic assumption in this work has been that the observed correlation $W\left(\lambda_{\max }\right)$ is caused primarily by a systematic change in the size distribution of interstellar grains and the consequent effects on alignment. Undoubtedly, the observational results are produced by particular combinations of all of the important parameters, such as size, optical properties, shape, alignment efficiency, and alignment direction. However, if these were to act independently, we would expect much more of a "scatter" diagram for $W\left(\lambda_{\max }\right)$. Some of the scatter in the present data may indeed be attributed to such "random" variations. A systematic variation in the optical properties of the grains, i.e., in material composition, with increasing (single) grain size, is difficult to imagine without a physical basis such as accretion of a mantle, as already treated here. Also, single-sized grains will give a much narrower polarization curve for the small values of $\lambda_{\max }$ than is shown by the observations, for any reasonable value of the dielectric refractive index. However, the possibility that chemical reactions on grain surfaces may alter the optical constants enough to cause the observed variations (Duley 1980) remains to be investigated.

A systematic variation of grain shape with grain size may possibly explain the variation in the data, but since finite nonspherical particles have wider polarization curves than infinite cylinder particles, it would be required that the grains increase in elongation as they grow larger. Although such "whisker" growth is possible, the case of simple accretion generally causes a grain to become more spherical, and $W$ would increase with $\lambda_{\max }$, opposite to the variation seen in the data for the smaller $\lambda_{\max }$. Such a shape effect could possibly contribute to an additional increase in width for the larger values of $\lambda_{\max }$. Since the influence of shape on the polarization curve appears to be small (Rogers and Martin 1979) and is not likely, by itself, to explain the observed variations in width, we neglect it here. Calculations for a size distribution of finite, nonspherical particles would be needed before more quantitative comparisons can be made.

Systematic variations in alignment efficiency, for a constant size distribution, can only couple large values of $\lambda_{\max }$ with larger widths, contrary to the trend in the data. Finally, if the data points were samples from regions of different inclinations between the line of sight and the magnetic field, $W$ should again be larger for larger $\lambda_{\max }$ (see Fig. 3 in Hong and Greenberg 1980). It appears that the only mechanism that will, by itself, reproduce the observed decrease in $W\left(\lambda_{\max }\right)$ for small grains is the modification of the size distribution by size-independent accretion. Furthermore, only a relatively steep initial size distribution (such as a power law) will show the effect. For example, the shape of an exponential size distribution with a size parameter greater than or about the total mantle thickness will not be as much affected by the accretion process, and $W$ will be an approximately constant function of $\lambda_{\max }$.

It should be emphasized that our results do not depend on where or when the mantles were accreted, as long as the mantle destruction probability is propor- 
tional to grain area $\left(\operatorname{size}^{2}\right)$. The $W-\lambda_{\max }$ relation does not distinguish between grains growing or eroding since the size distribution is a unique function of $\Delta a$, independent of its direction of change. The SSA case, perhaps, should be excluded. If a destructive process (e.g., photon sputtering) erodes mantles that are presumed to have accreted under very dense conditions and it also destroys the surface "rockets" faster than it uncovers a 3.7 $\AA$ molecular layer, the grains would lose their suprathermal advantage. Both $W$ and $\lambda_{\max }$ would be much larger for the same $\Delta a$ than when the mantle accreted and the "rockets" were protected. For example, in the $W-\lambda_{\max }$ plane the grains would tend to move from the lowest SSA curve in Figure 6 to the upper TSA curve in Figure 5 and follow that curve "backward" as the grains were denuded of their mantles.

The question of mantle accretion is intimately tied to the question of heavy element abundances. The mantle thicknesses assumed in this work can only derive from the most common elements $\mathrm{O}, \mathrm{N}$, and $\mathrm{C}$, and we have allowed $72 \%$ of the total cosmic abundance of these elements to be "accretable" (Greenberg 1982). For our size distributions, the amount of elements heavier than He that is tied up in the core grains is $\sim 10 \%$ of the cosmic abundance of all the heavy elements, and consistent with the fact that most interstellar elements other than $\mathrm{O}, \mathrm{N}$, and $\mathrm{C}$ are heavily depleted under most circumstances. The situation for $\mathrm{O}, \mathrm{N}$, and $\mathrm{C}$ is much less clear. In the diffuse interstellar medium, carbon appears depleted by a factor of as much as 5 (Savage and Mathis 1979), while nitrogen is depleted by about a factor of 2 (Lugger et al. 1978). According to de Boer (1979) oxygen is depleted by less than a factor of 2 , although recently Martin and York (1982) found a depletion by a factor of 5 to 6 in an unionized cloud that was also observed by de Boer. The appearance of the $3.1 \mu \mathrm{m}$ ice band in some heavily reddened stars definitely implies depletion of oxygen under very dense circumstances. The lack of, or the insignificance of, the ice feature in intermediate clouds may have been caused by processing of a portion of the mantle material into some other compound than primarily $\mathrm{H}_{2} \mathrm{O}$ ice (Greenberg 1978; 1982). In any case, the amount of depletion of accretable material in Figures 4-6 is less than or about a factor of 2 for $\lambda_{\max }<0.6 \mu \mathrm{m}$, consistent with the depletion data for the diffuse medium. Determination of accurate heavy element abundances in regions where $\lambda_{\max }>0.6 \mu \mathrm{m}$ would provide an additional test for the model proposed here.

If the grain size distribution systematically varies from region to region owing to mantle accretiondestruction processes, it appears from our results that the thermal alignment mechanism (Davis and Greenstein 1951) fails to explain the observed polarization widths at large values of $\lambda_{\max }$. Even with a magnetic enhancement factor of the order of 100 or more that may explain the polarization at values of $\lambda_{\max }$ for the diffuse clouds, the increasing disalignment when the grains grow larger leads to a rapid increase in $W$ with $\lambda_{\max }$ which is not consistent with the data. The only way to reproduce the smaller widths seen at the larger $\lambda_{\max }$ would be to assume that the magnetic enhancement factors are consistently larger with larger grains, which has no obvious physical justification. Of course, the assumption of a particular magnetic enhancement factor in the first place is already rather arbitrary, and has been resorted to as a means of bringing the inefficient TSA mechanism into better agreement with the observed polarizing efficiency of the grains at visual wavelengths. Since this remedy fails to explain the trends in the present data, we conclude that thermal alignment is inadequate as a mechanism for orienting interstellar grains.

Although thermal alignment fails, it is gratifying that the other mechanism considered here, the Purcell's SSA mechanism when limited by mantle accretion, is capable of explaining the observed correlation of $W$ with $\lambda_{\max }$. In fact, with the exception of the one high data point at $\lambda_{\max }=0.47 \mu \mathrm{m}$, the predicted variation in Figure 6 for enhancement factors of the order of unity agrees remarkably well with the data points, including both the predicted minimum at $\lambda_{\text {max }} \approx 0.5 \mu \mathrm{m}$ and the predicted maximum at $\lambda_{\max } \approx 0.7 \mu \mathrm{m}$. However, more accurate observations in this region are clearly needed. It is worth emphasizing that the SSA mechanism coupled with growth through depletion offers a natural way of explaining efficient alignment where the grains are large $\left(\lambda_{\max }>0.7 \mu \mathrm{m}\right)$, as sometimes observed (Johnson 1982). Furthermore, it is then a natural consequence of this model that the presence of icy mantles should be correlated with strong polarization, as has been found for compact infrared sources associated with dense molecular clouds (Joyce and Simon 1982).

One possible difficulty for the SSA mechanism may lie in the ratio of the correlation time to the damping time (eq. [11]) which must be greater than unity. For our choice of parameters, $t_{L} / t_{d} \approx 5.6[0.1 \mu \mathrm{m} / a(\mu \mathrm{m})]$, which seems adequate for all but the largest grains. However, when the accretion process first starts on a grain, this ratio may be decreased by about a factor of 3 (the ratio of the density of the core material to the density of the accreted layers), and $t_{L} / t_{d}$ is then of the order of unity for grain sizes $\sim 0.2 \mu \mathrm{m}$. On the other hand, a $0.2 \mu \mathrm{m}$ grain is the largest grain in the initial size distribution, and our results here are therefore not much affected by this consideration. It is perhaps more uncertain whether our choice of a "correlation thickness" equal to a typical molecular diameter is correct, but it would be surprising if it was much smaller. Such details clearly depend on the nature of the "driving" process for the "rockets" and these remain to be worked out. 
We have not considered the ferromagnetic or superparamagnetic mixed oxide grains proposed by Duley (1978) as a way to orient core-mantle grains. It appears that for this mechanism to do better than, for example, the Davis-Greenstein thermal alignment mechanism, the effective total grain, and core grain sizes must be larger than those we have been treating here.

An important observational parameter is the polarizing efficiency of interstellar grains as measured by the ratio of polarization to color excess. Its maximum value in the diffuse cloud medium is $\sim 0.09$ (Greenberg 1978; Fig. 2.7). The values calculated here for the grains with $\lambda_{\max }=0.5-0.7 \mu \mathrm{m}$ (mantle size 0.06-0.1 $\mu \mathrm{m}$ ) for the SSA case with no magnetic enhancement is $0.13-0.08$, slightly higher than the observations. A decrease in these values owing to a less than optimal orientation of the magnetic field or a grain component that contributes to extinction and not to polarization may therefore still give a ratio consistent with the observations. The polarization-to-extinction ratio has also been observed to decrease with increasing optical depth (Vrba et al. 1981), consistent with the trend for moderate size grains $\left(\lambda_{\max }\right.$ $=0.5-0.7 \mu \mathrm{m})$ in this work. Another observational quantity of interest is the ratio $R$ of visual extinction to color excess and its well defined correlation with $\lambda_{\max }$ $\left(R \approx 5.8 \lambda_{\max } ;\right.$ Savage and Mathis 1979). All grain components, however, must be included before quantitative comparisons can be made between our model and these data.

Although Vrba, Coyne, and Tapia (1981) find that a combination of grain growth and increasing gas density is responsible for the reduction of grain alignment with optical depth in the outer regions of the RCrA cloud, they argue that accretion onto grains is not likely to be the main source of grain growth. The grains would apparently not grow mantles fast enough to explain their observations. However, their argument is based upon assuming that the sticking coefficient is unlikely to be larger than 0.2 and that the age of the cloud is given by the ages of the stars. We consider a sticking coefficient of unity quite likely for such cold and partially shielded grains, in which case the required cloud age is $2 \times 10^{6} \mathrm{yr}$, which is quite reasonable for stars that are $<10^{6} \mathrm{yr}$ old. Also, the procedure used in deriving the limit on the accretion time scale assumes a circular cross section for the cloud, so that the unknown geometry may also play a role. One interesting feature of their observations is the apparent wider size distribution of grains with increasing optical depth, as inferred from the increasing dispersion in $\lambda_{\max }$-values, in contradiction to what is predicted by the model here. However, it is quite likely that in looking through this cloud one is sampling regions of differing densities, or a density gradient, and a larger variety is sampled the closer one looks to the central positions. If so, one should expect a larger spread in $\lambda_{\max }$ values than for the diffuse medium, even though the size distribution in any particular location is narrower than in the diffuse medium. Detailed observations of the width of the polarization curve would be very interesting, and would test the predictions in this model.

The growth mechanism favored by Vrba, Coyne, and Tapia (1981) to explain the RCrA cloud observations is grain coagulation. This is also the mechanism invoked by Mathis and Wallenhorst (1981) to explain the peculiar extinction curves for the graphite-silicate grain mixture, and was proposed earlier by Jura (1980) as an explanation for the large value of the ratio of column density of hydrogen to color excess seen in the direction of $\rho \mathrm{Oph}$. However, the $\rho$ Oph observation cannot be explained by the graphite-silicate mixture, and may be caused by systematic effects in the hydrogen column density (Mathis and Wallenhorst 1981). We have shown here that coagulation by turbulent collisions of grains is not likely to explain the total observed variation in $W$ with $\lambda_{\text {max }}$, although it could perhaps contribute to the observed variation for moderate values of $\lambda_{\max }$. The time scale $t_{c}$ is $\sim 10^{7} \mathrm{yr}$, assuming a dust to hydrogen number ratio of $\sim 10^{-12}$, a hydrogen density of $\sim 10^{4} \mathrm{~cm}^{-3}$, and a gas rms turbulent velocity of $\sim 0.5 \mathrm{~km} \mathrm{~s}^{-1}$. Since significant changes in the size distribution are present at $\sim 0.1 t_{c}$ (Fig. 3), coagulation may play a role in modifying grain sizes under dense conditions. Of course, our inability to treat the supersonic motions clearly present in many interstellar clouds makes such conclusions quite uncertain. On the other hand, coagulation driven by radiation or diffusion in a gravitational and a magnetic field lead to similar or even smaller time scales (Scalo 1977). We would expect the effect on the size distribution to be essentially the same as for turbulent coagulation. It is, however, difficult to see how such grains would be more effectively aligned as they grow larger, as seems implied by the data. They could perhaps be preferentially located where the "rocket" process is particularly effective, for example, near shock fronts as suggested by Johnson et al. (1981) for the Orion Nebula, but large-scale evidence for this is presently lacking.

Rather than coagulating in dense regions, core-mantle grains may have their mantles explosively removed in grain-grain collisions (Greenberg 1979). According to d'Hendecourt et al. (1982) irradiated mantles with frozen radicals present at the $1 \%$ level or more will lead to an explosive release of a large part of the mantle material if the grain is suddenly heated to $27 \mathrm{~K}$ or higher. This corresponds to a critical collision velocity of $\sim 0.04$ $\mathrm{km} \mathrm{s}^{-1}$, an order of magnitude lower than required for shattering and less than the turbulent velocities considered earlier. If this process is occurring, it may solve the long-standing puzzle of why all heavier gas components are not frozen out on the grains in the dense clouds. The argument is limited by the fact that the cloud density cannot be much higher than $10^{4} \mathrm{~cm}^{-3}$ where photo- 
processing may be too inefficient (unless internal sources are assumed) and that it cannot be much less than $10^{3}$ $\mathrm{cm}^{-3}$ if the grain collision time scale is not to be larger than cloud lifetimes of a few $10^{7} \mathrm{yr}$. However, for densities of $10^{3}-10^{4} \mathrm{~cm}^{-3}$, mantle explosions could influence the grain size distribution and therefore the predictions we have made here.

We have not inquired into the detailed composition of the mantle, except to require it to be a dielectric with a refractive index characteristic of ice. In the evolutionary grain model of Greenberg (1982) nonvolatile mantles are present on core grains in the diffuse interstellar clouds. The mean grain size is $\sim 0.12 \mu \mathrm{m}$, which for our size distribution would correspond to a mantle thickness of $0.06 \mu \mathrm{m}$ with $6 \%$ of the accretable material being depleted. For the SSA case with no magnetic enhancement, this distribution leads to $\lambda_{\max }=0.56 \mu \mathrm{m}$ and $W=1.76$, in reasonable agreement with diffuse cloud observations. We may therefore consider the smaller values of $\lambda_{\max }$ as brought about by the removal or nonpresence of even the nonvolatile mantle, and the larger $\lambda_{\max }$-values brought about by the presence of an increasingly larger (partially or wholly volatile) additional mantle acquired under denser conditions. Such a core-mantle-mantle model would seem to be able to offer a consistent picture of many observational grain characteristics. Extinction calculations for such a complex particle are being investigated.

We have experimented a bit with different refractive indices. In general, $\lambda_{\max }$ and $W$ will both increase with increasing $\mathrm{m}^{\prime}$ for either the core or the mantle material. However, if the nonvolatile mantle has an index of, for example, $m^{\prime}=1.45, \lambda_{\max }$ increases by less than $30 \%$ compared to the $m^{\prime}=1.3$ "icy" case, if the mantle thickness is less than $0.07 \mu \mathrm{m}$ (assuming PSA). We expect therefore that the core-mantle-mantle grains referred to above would lead to $W$ versus $\lambda_{\max }$ curves slightly shifted to the right and upward, but shifted with lesser amounts for larger $\lambda_{\text {max }}$, because of the increasing dominance of the volatile mantle. The large-scale shape of the curves, therefore, would appear to be very much the same.

\section{CONCLUSIONS}

The observed correlation of the width of the polarization curve with wavelength of maximum polarization is most likely caused by a systematic variation in the size distribution of interstellar grains. Size modification of a power-law distribution by coagulation of grains seems unlikely to explain the present data, as shown here for the case of subsonic turbulent coagulation. However, modification of the grain sizes by accretion of dielectric mantles reproduces quantitatively the observed correlation. Our infinite cylinder calculations show that the data imply imperfect spinning alignment for the grains, but also that the Davis-Greenstein thermal alignment mechanism is too inefficient in aligning the grains when they have acquired large mantles. On the other hand, calculations assuming Purcell's suprathermal alignment mechanism with the "rocket" lifetime determined by a monolayer accretion time scale give results consistent with the observed data. We have shown that this mechanism leads to a width versus $\lambda_{\text {max }}$ relation characterized by (1) an initial steep decrease in width with increasing $\lambda_{\max }$ caused by the rapid narrowing of the size distribution from mantle accretion, (2) an increase in width with intermediate values of $\lambda_{\max }$ because of the increasing disalignment as the grains grow larger, and (3) another decrease in width for the largest values of $\lambda_{\max }$ when the increasing depletion of accreting material makes the accretion time scale long enough to more than compensate for the disalignment due to size. However, the details of the "rocket" process in the presence of mantle accretion remain to be worked out. Also, more accurate observations for a larger number of sources are desirable to further test the predictions of the model presented here.

P. A. A. wishes to thank the Netherlands Organization for the Advancement of Pure Research (ZWO) for support during this work. He is also grateful to the Huygens Laboratorium for extending its hospitality and use of facilities and computer time. Support by the Nato Science Program through a Senior Fellowship to P. A. A. is also gratefully acknowledged.
Aannestad, P. A. 1982, Astr. Ap., 115, 219

Aannestad, P. A., and Kenyon, S. J. 1979, Ap. J., 230, 771.

Allen, M., and Robinson, G. W. 1975, Ap. J., 195, 81.

Biermann, P., and Harwit, M. 1980, Ap. J. (Letters), 241, L105.

Codina-Landaberry, S., and Magalhaes, A. M. 1976, Astr. Ap., 49, 407.

Coyne, G. V., Gehrels, T., and Serkowski, K. 1974, A.J., 79, 581

Davis, L., and Greenstein, J. L. 1951, Ap. J., 114, 206.

de Boer, K. S. 1979, Ap. J., 229, 132.

d'Hendecourt, L. B., Allamandola, L. J., Baas, F., and Greenberg,

J. M. 1982, Astr. Ap., 109, L12.

Duley, W. W. 1978, Ap. J. (Letters), 219, L129. 1980, Ap. J., 240, 950.

Greenberg, J. M. 1968, in Stars and Stellar Systems, Vol. 7, Nebulae and Interstellar Matter, ed. B. M. Middlehurst and L. A. Aller (Chicago: University of Chicago Press), p. 221.

\section{REFERENCES}

Greenberg, J. M. 1969, Physica, 41, 67.

1971, Astr. Ap., 12, 240.

1978, in Cosmic Dust, ed. J. A. M. McDonnel (New York:

Wiley-Interscience), p. 187.

1979, in Stars and Star Systems, ed. B. E. Westerlund

(Dordrecht: Reidel), p. 173.

1982, in Submillimetre Wave Astronomy, ed. J. E.

Beckman and J. P. Phillips (Cambridge: Cambridge University Press), p. 261.

Greenberg, J. M., and Chlewicki, G. 1983, Ap. J., in press.

Greenberg, J. M., and Hong, S. S. 1974, in IAU Symposium 60

Galactic Radio Astronomy, ed. F. J. Kerr and S. C. Simonson III

(Dordrecht: Reidel), p. 155.

Hong, S. S., and Greenberg, J. M. 1980, Astr. Ap., 88, 194.

Johnson, P. E. 1982, Nature, 295, 371. 
Johnson, P. E., Rieke, G. H., Lebofsky, M. J., and Kemp. J. C. 1981, Ap. J., 245, 871 .

Jones, R. V., and Spitzer, L., Jr. 1967, Ap. J., 147, 943.

Joyce, R. R., and Simon, T. 1982, Ap. J., 260, 604.

Jura, M. 1980, Ap.J., 235, 63.

Lugger, P. M., York, D. G., Blanchard, T., and Morton, D. C. 1978, Ap. J., 224, 1059.

Martin, E. R., and York, D. G. 1982, Ap. J., 257, 135.

Martin, P. G. 1973, in IAU Symposium 52, Interstellar Dust and Related Topics, ed. J. M. Greenberg and H. C. van de Hulst (Dordrecht: Reidel), p. 161.

Mathis, J. S. 1979, Ap. J., 232, 747.

Mathis, J. S., Rumpl, W., and Nordsieck, K. H. 1977, $A p . J ., 217$, 425.

Mathis, J. S., and Wallenhorst, S. G. 1981, Ap. J., 244, 483.

Mouschovias, T. Ch. 1978, in Protostars and Planets, ed. T. Gehrels (Tucson: University of Arizona Press), p. 209.
Onaka, T. 1980, Ann. Tokyo Astr. Obs., 18, 54.

Purcell, E. M. 1976, $A$ p. J., 206, 685 1979, Ap. J. 231, 404.

Purcell, E. M., and Spitzer, L., Jr. 1971, Ap. J., 167, 31.

Rogers, C., and Martin, P. G. 1979, Ap. J., 228, 450.

Savage, B. D., and Mathis, J. G. 1979, Ann. Rev. Astr. Ap., 17, 73 Scalo, J. M. 1977, Astr. Ap. 55, 253.

Serkowski, K. 1973, in IAU Symposium 52, Interstellar Dust and Related Topics, ed. J. M. Greenberg and H. C. van de Hulst (Dordrecht: Reidel), p. 145.

Shapiro, P. R. 1975, Ap. J., 201, 151.

Spitzer, L., Jr., and McGlynn, T. A. 1979, Ap. J., 231, 417.

Vrba, F. J., Coyne, G. V., and Tapia, S. 1981, Ap. J., 243, 489.

Völk, H. J., Jones, F. C., Morfill, G. E., and Röser, S. 1980, Astr. Ap., 85, 316

Wilking, B. A., Lebofsky, M. J., Martin, P. G., Rieke, G. H., and Kemp, J. C. 1980, Ap. J., 235, 905.

P. A. Aannestad: Department of Physics, Arizona State University, Tempe, AZ 85287

J. M. Greenberg: Laboratory Astrophysics, University of Leiden, PO Box 9504, 2300 RA Leiden, the Netherlands 